\title{
Cardiovascular disease as a leading cause of death: how are pharmacists getting involved?
}

This article was published in the following Dove Press journal: Integrated Pharmacy Research and Practice

\section{Kevin Mc Namara ${ }^{1-3}$ \\ Hamzah Alzubaidi ${ }^{4}$ \\ John Keith Jackson ${ }^{3}$}

'School of Medicine, Deakin University, Geelong, VIC, Australia;

${ }^{2}$ Deakin Health Economics, Centre for Population Health, Deakin University, Geelong, VIC, Australia; ${ }^{3}$ Centre for Medicine Use \& Safety, Faculty of Pharmacy \& Pharmaceutical Sciences, Monash University, Melbourne, VIC, Australia; ${ }^{4}$ nstitute for Medical Research and College of Pharmacy, University of Sharjah, Sharjah, Sharjah, United Arab Emirates
Correspondence: Kevin Mc Namara School of Medicine and Centre for Population Health, Deakin University, 75 Pigdons Road, Waurn Ponds, Geelong, VIC 3216, Australia

Tel +6I 352479472

Email kevin.mcnamara@deakin.edu.au

\begin{abstract}
Cardiovascular diseases (CVDs) are a leading cause of death globally. This article explores the evidence surrounding community pharmacist interventions to reduce cardiovascular events and related mortality and to improve the management of CVD risk factors. We summarize a range of systematic reviews and leading randomized controlled trials and provide critical appraisal. Major observations are that very few trials directly measure clinical outcomes, potentially owing to a range of challenges in this regard. By contrast, there is an extensive, high-quality evidence to suggest that improvements can be achieved for key CVD risk factors such as hypertension, dyslipidemia, tobacco use, and elevated hemoglobin A1c. The heterogeneity of interventions tested and considerable variation of the context under which implementation occurred suggest that caution is warranted in the interpretation of meta-analyses. It is highly important to generate evidence for pharmacist interventions in developing countries where a majority of the global CVD burden will be experienced in the near future. A growing capacity for clinical registry trials and data linkage might allow future research to collect clinical outcomes data more often. Keywords: health services, chronic disease management, cardiovascular risk factors, preventative health, disease screening
\end{abstract}

\section{Current trends in cardiovascular disease}

Cardiovascular diseases (CVDs) are a leading cause of mortality internationally. When combined, ischemic heart disease and all forms of stroke were the attributed causes of death for an estimated 13 million people globally in 2010, a quarter of the global totally (increased from just one in five deaths 20 years earlier). ${ }^{1}$ This increase hides a considerable variation in trends and in epidemiology for different regions of the world As a general rule, developed regions such as Western Europe, North America, and Australia/ New Zealand are considered to be in the fourth (highly advanced) stage of what is known as the "epidemiological transition" of health burden. ${ }^{2}$ From a cardiovascular (CV) point of view, epidemiological transition suggests that developed societies will experience a reduction in infection-related conditions such as rheumatic heart disease and nutrition deficiency-induced conditions affecting heart muscle. Conversely, in emerging economies, there is a pronounced increase in age-adjusted incidence of noncommunicable diseases such as CVD induced by changing lifestyles and increasing life expectancies. ${ }^{2}$

This epidemiological transition evolves alongside various social and economic developments - the fourth stage typically produces a decline in CVD incidence as countries implement systems to better prevent and treat CV conditions. Age-adjusted mortality rates attributed to CVD have declined consistently and considerably over 
the past three or four decades in developed countries, ${ }^{3}$ perhaps creating some complacency that the challenge is over. However, the decline in crude mortality rates has tailed off in recent years as an aging society and increased prevalence of obesity and diabetes offset the gains made in other areas. ${ }^{3}$ Repeated population surveys identify the considerable evidence-treatment gaps for common CVD risk factors such as hypertension and high cholesterol. ${ }^{4-6}$ Health services in many developed countries are now bracing themselves not just only for anticipated increases in obesity and diabetes as the population ages but also for related rises in conditions such as atrial fibrillation, heart failure, and chronic kidney disease. $^{7-9}$

There are two general approaches to reducing the CVD burden, which can operate in concert with each other. The first is to apply measures across the community with the aim of encouraging population-wide shifts toward healthier behaviors. Such initiatives are often at a policy level and include initiatives such as taxes on sugar-sweetened drinks or tobacco, health advertising, or enabling active transport options such as cycle lanes in local communities. Health professionals working in clinical settings tend to focus most of their efforts on the second approach, which involves identifying and working with elevated-risk individuals to prevent or manage CVD, its risk factors, and its complications. At the earliest stages, this involves active screening for risk of disease so that any elevated risk can be addressed early. For an established risk of disease, prevention and management in primary care are multifaceted, but will typically involve lifestyle modification support and, where appropriate, the use of medications.

The focus of this article is to consider the evidence base for community pharmacy interventions in the context of contemporary care to reduce the impact of CVD mortality. Such a role in disease management for CVD is well established internationally for hospital pharmacists, but formalizing such a role of primary-care pharmacists (PCPs), including community pharmacists, is still evolving. There is a clear logic to the involvement of community pharmacists. They offer ready access to care through their convenient locations and opportunistic interventions for those with elevated CVD risk or established CVD. ${ }^{10}$ Unlike many health professionals, they have extensive medicines' knowledge and can provide a holistic view of therapy. As the population with CVD becomes older, care becomes fragmented and uncoordinated while the presence of comorbidities makes treatment decisions more complex. ${ }^{11}$ The broad knowledge of medication that pharmacists possess appears to position the professional particularly well to provide critical input to issues such as explaining treatment options, drug-drug interactions, and dosage adjustment and to initiate shared decision-making around therapy initiation and even deprescribing in some patients. ${ }^{11,12}$

\section{Methods}

We first conducted a brief scoping search for systematic reviews in EMBASE using relevant terms to explore the nature and extent of evidence for community pharmacist interventions to reduce CVD mortality. This literature search was performed to supplement the authors' existing knowledge of the key literature. The EMBASE review combined the results of three searches that used appropriate terms to identify studies of 1) common CVD risk factors, established CVDs, health promotion, and common CV events; 2) pharmacist and pharmacy services (clinical or community); and 3 ) terms that describe a review of existing literature. Specific search terms used are outlined in Box 1 (Search 1). Hospital and clinical pharmacist search terms were included. Although such pharmacy services were not the focus of the search, they are frequently combined with community pharmacy services in systematic reviews, and it was uncertain whether clinical pharmacists might be performing relevant duties in a primary care setting. Published reviews were examined in detail if pharmacist interventions were the key focus, or if the abstract identified the inclusion of pharmacist studies. While we searched for systematic reviews, we also considered findings from other relevant literature reviews in our search results. As this article was not designed as a formal systematic review, we did not apply quality filters to the articles. Rather, we sought to describe all of the relevant literature identified so that important shortcomings in the available evidence base could also be described. A brief search for economic evaluations of pharmacist interventions with a CV component was added (Box 1, Search 2).

\section{Overview of the literature}

Most CVD-specific systematic reviews identified in this area related to pharmacist interventions for CVD risk factors, diabetes, heart failure, anticoagulation, and secondary prevention generally. We also explored systematic reviews that evaluated general medication management services by pharmacists for CVD-related outcomes, acknowledging that many pharmacist services for people with CVD are delivered under such a framework. We briefly summarized the evidence around hospital pharmacists as part of this literature overview for several reasons. While there are obvious differences in 
Box I EMBASE search strategy used to support the review

\begin{tabular}{|ll|}
\hline Search I & \\
I.I - Cardiovascular/intervention & Cardiovascular disease \\
or subject heading) & Cardiovascular disease \\
& assessment \\
& Hypertension \\
& Hyperlipidemia \\
& Chronic kidney failure \\
& Kidney disease \\
& Atrial fibrillation \\
& Smoking cessation \\
& Diabetes mellitus \\
& Heart failure \\
& Acute coronary syndrome \\
& Cerebrovascular disease \\
& Angina pectoris \\
& Health promotion \\
I.2 - Pharmacist search terms (use of & Pharmacy \\
any of these terms in title or abstract) & Clinical pharmacy \\
& Pharmacist \\
& Pharmacist intervention \\
Combine I.I AND 2.I AND 2.2 & Hospital pharmacy \\
Search 2 & Hospital pharmacy service \\
2.1 - Health economic terms & Hospital pharmacist \\
I.3 - Systematic review terms (use of & Systematic review \\
any of these terms in title or abstract) & Literature review \\
& Meta-analysis \\
& \\
& Cost-benefit analysis \\
& Cost-effectiveness analysis \\
& Economic impact \\
& Clinical pharmacy \\
& Pharmacist \\
& \\
& \\
&
\end{tabular}

the role and the clinical environment between settings, presenting hospital-based evidence does offer some indication of the intrinsic potential of pharmacists to deliver relevant interventions. It also offers some insight as to the relative evidence base for hospital versus community pharmacy in delivering these interventions.

\section{Evidence of mortality reduction through CVD risk factor management}

Several systematic reviews examined the impact of pharmacist interventions relating specifically to hypertension, dyslipidemia, smoking cessation, and sleep apnea, alongside a review of interventions for CVD risk factors more generally.

Hypertension was the most commonly reviewed risk factor, reflecting the large number of pharmacist studies in this area and prevalence of the condition. Intervention scope varied and included disease management, medication management, and medication adherence interventions. None of the reviews examined the impact on mortality or $\mathrm{CV}$ events, a reflection of the general absence of these data from trials. Equally, while reviews generally found a positive impact from pharmacist intervention, meta-analysis often did not examine the specific overall effect of community pharmacists, as distinct from pharmacists intervening in other settings. ${ }^{13-16}$ However, based on systematic reviews, there is a reasonable evidence to suggest a positive impact of pharmacist interventions on blood pressure (see next section).

Just like for hypertension, systematic reviews of community pharmacy services for CVD and diabetes risk screening, diabetes, obstructive sleep apnea, and management of hyperlipidemia all suggest a positive impact, but with a focus on outcomes that related to the process of care rather than to clinically relevant endpoints. For example, in reviews of patients with diabetes receiving pharmacist interventions, extensive evidence is documented of significant improvements in hemoglobin A1c (HbA1c), a key measure of diabetes control, as well as medication adherence, patient knowledge, and other CVD risk factors such as cholesterol and blood pressure. ${ }^{17-23}$ The Asheville Project demonstrates the potential to implement complex interventions and produce meaningful, long-term improvements in risk factors such as HbAlc. ${ }^{24}$ Clifford et al demonstrated significant reductions in both $\mathrm{HbA1c}$ and, using a validated risk algorithm, median 10-year risk of CVD and stroke onset (from $26.1 \%$ to $20.3 \%$ in the intervention group) in patients who received pharmaceutical care, compared with control group participants whose CVD risk slightly increased. ${ }^{25}$ Moreover, an independent positive effect was observed from pharmaceutical care that was above and beyond the benefits of therapy changes. However, evidence that the interventions above can deliver a prospectively assessed and meaningful reduction in hard endpoints such as CV events, CVD onset, and CV-related mortality associated with such interventions appears to be missing. ${ }^{17-22}$ This apparent absence of clinical endpoints mirrors the conclusion of a recent systematic review examining the impact of all pharmacist interventions and which examined CVD outcomes, all-cause mortality, and other outcomes. ${ }^{26}$ There is also commentary in the systematic review literature about the need to improve the general quality of pharmacist studies in this area. ${ }^{18,27}$

The Canadian Cardiovascular Health Awareness Program study is a notable exception to the limitations of evidence cited above. ${ }^{28}$ This was a well-conducted cluster randomized 
controlled trial (RCT) where communities in the intervention arm, with volunteers working in conjunction with pharmacists to deliver pharmacy screening for hypertension, experienced a $9 \%$ reduction in cardiac admissions compared with communities who received usual care. This study was conducted using discrete rural communities as clusters to overcome the challenge of intervention contamination. This approach allowed changes in cardiac admission rates for intervention versus control communities to be reasonably attributed to the intervention owing to the relatively isolated health system from a data perspective. The obvious shortcoming of relying on this population approach to outcomes data is the inability to test effectiveness in urban settings where the context for care might be different. For example, if treatment is received in different localities to patients' residence, or the hospital where they would be admitted for a cardiac event, intervention contamination and dilution of effect are risks. Rapidly advancing data-linkage technology now provides the opportunity to track individuals who receive pharmacist care so that long-term outcomes can be established at an individual patient level. This would allow a more compelling case to be established around the impact of pharmacist care to reduce CVD risk. The feasibility of data linkage - using patient identifiers to link local patient care records with health outcome information on other databases (eg, mortality registers) - might enable the tracking of outcomes following pharmacist intervention. This has been demonstrated in some settings already, eg, by Roughead et al who demonstrated significantly reduced rates of hospitalization due to bleeding among veterans who were prescribed anticoagulants, among those who received medication review by pharmacists compared with those who did not. ${ }^{29}$

\section{Evidence of mortality benefit for patients with established CVD}

A smaller number of reviews appear to have been conducted which examine the role of community pharmacists in the management of established heart disease, when compared with reviews of community pharmacist interventions for risk factors including diabetes. Interestingly, Altowaijri et al's review of clinical pharmacist interventions for CVD in any setting found six trials examining mortality benefits from pharmacist interventions; ${ }^{30}$ four of the six studies reported to demonstrate positive mortality or disease control outcomes were all focused on outpatients, whereas the other two were community pharmacy studies. It is important to note that these community pharmacist interventions were described as education-only in nature and did not involve active medication management roles as many of the hospital studies did. ${ }^{30}$ Observed in closer detail, the first study was effectively a diuretic treatment adherence study from the Netherlands involving 152 patients with heart failure who were randomized to monthly pharmacist interviews for 6 months or usual care. ${ }^{31}$ In addition to having a relatively narrow scope for intervention, the study was only powered to detect its primary outcome, noncompliance, and so the absence of significant improvements for secondary clinical endpoints, should be interpreted in the context of the trial's scope.

The other trial was the MEDMAN RCT from the UK involving 293 patients with heart failure. ${ }^{32}$ The intervention involved pharmacists providing medication review and lifestyle modification support over two visits to each patient's home. The trial outcomes provided no evidence to suggest improvements in hospitalization rates at 6 months (the primary outcome) or mortality. Conversely, a nonsignificant increase in mortality and health service use was observed in the intervention group. There are a number of factors that could explain the absence of a positive effect. Notable among these is the fact that only half of the intervention pharmacist recommendations were acted upon by the patient's general practitioner (GP), while health behaviors were perhaps better than expected at baseline and rendered lifestyle modification advice largely unnecessary (eg, only six of 149 intervention patients smoked, most patients did not drink alcohol). As such, the impact of the intervention on treatment may have been less than expected.

The greater ability of hospital-based pharmacists, acting in multidisciplinary teams, to ensure follow-through on therapy recommendations may help to explain the positive results observed in that setting. Other reviews confirm a shortage of "hard" endpoints in relevant community pharmacist trials. ${ }^{33,34}$ An extensive 2016 review of 26 pharmacist interventions for heart failure and acute coronary syndrome confirmed a continuing lack of high-quality trials with hard endpoints, although it did also report the Heart failure and Optimal Outcomes from Pharmacy Study trial in primary care. ${ }^{35}$ This trial randomized 87 practices with 1,090 eligible patients with left ventricular systolic dysfunction, to receive PCP intervention or usual care. No significant differences were observed between groups in the primary outcome, the composite of death from any cause or hospital admission for worsening heart failure, or other hard endpoints.

Warfarin management is another area where there is compelling outcome evidence in support of clinical pharmacy services compared with usual medical management. ${ }^{36,37}$ Meta-analyses suggest reduced rates of major bleeds and 
potentially reduced rates of thromboembolic events ${ }^{36,37}$ and also high rates of time in therapeutic range relative to usual care. Correspondingly, individual studies examining community pharmacist interventions seem to indicate the potential for high-quality care and impressive international normalized ratio (INR) control - eg, a pre-post intervention study by Harrison et al found that time within therapeutic INR range increased significantly when anticoagulant management was switched from GPs (62\%) to pharmacists (79\%) ${ }^{38}$ However, we are unaware of any attempts to examine the impact on clinical outcomes of switching to pharmacist-led care. We were also unable to identify any systematic review of community pharmacist interventions for the management of stroke, kidney disease, and peripheral vascular disease or the prevention of complications associated with CVD. ${ }^{39}$ This is despite often relatively extensive studies and relevant systematic reviews to determine the efficacy of similar interventions by clinical pharmacists. ${ }^{40}$

\section{Evidence of intermediate health gains from community pharmacist interventions}

The absence of extensive evidence around mortality benefits from community pharmacist interventions needs to be considered in light of the other available evidence - lack of evidence of an effect is not the same as the absence of an effect. It may be explained by the relative difficulty of linking participant data in community pharmacy to $\mathrm{CV}$ and mortality outcomes until relatively recently in most countries and by inadequate research budgets and time frames which necessitate short-term and affordable outcomes such as adherence and risk factor measurements. There are compelling arguments in terms of disease detection and control of risk factors, alluded to above, which tend to suggest a strong potential for the reduction of CVD risk when considered in aggregate. This section summarizes some of the key evidence in this regard and considers other issues relevant to feasibility and acceptability.

\section{CVD risk factor monitoring and management}

Globally, community pharmacists have provided interventions aimed at reducing modifiable CVD risk factors including smoking cessation, weight loss, and control of blood pressure and lipid levels. The value of these interventions has been well documented in the literature.
The effectiveness and cost-effectiveness of communitypharmacist smoking cessation interventions have been suggested in several reviews. ${ }^{41-43}$ Blenkinsopp et al reviewed two RCTs and three nonrandomized experimental studies. ${ }^{41}$ In both RCTs, pharmacists underwent training in the Stages of Change model to help them offer evidence-based advice. Outcome measures included self-reported smoking cessation rates, but the time points differed in the two RCTs having significant differences in abstinence rates between the control and the intervention groups at 1 year ( $2.7 \%$ and $14.3 \%$, respectively). The smoking cessation rates and health economic analysis of the two trials demonstrated that smoking cessation programs in community pharmacies were effective and cost-effective.

A systematic review of smoking cessation trials involving pharmacist interventions by Brown et al included 12 studies, 10 of which were RCTs. ${ }^{43}$ Interventions included behavioral support, nicotine replacement therapy, and photoaging, while controls received usual care. Five of the 12 studies showed that the intervention had significant effects on improving abstinence rates, and the four studies that reported cost-effectiveness analysis found that the intervention was cost-effective. In the same review, Brown el at also looked at five studies on weight loss interventions in community pharmacies. All studies compared a weight loss intervention in the community pharmacy (combinations of meal replacement, low-calorie diet, exercise, and orlistat) with similar interventions in other primary care settings, commercial weight loss programs, or another intervention in the community pharmacy, ie, participants in both intervention and control arms received "active" interventions. Pharmacy interventions resulted in weight reductions similar to those obtained with active interventions in other healthcare settings. In terms of cost-effectiveness, however, pharmacy-based interventions were similar in cost to interventions in other healthcare settings and were more expensive than commercial weight loss programs. The duration of the intervention appeared to be a key factor determining the effectiveness of community-pharmacy-delivered weight loss and smoking cessation interventions, probably reflecting the benefits of ongoing monitoring, and support for ongoing maintenance and relapse prevention that extended interventions facilitate for health behavior change. ${ }^{44}$

Several RCTs and meta-analyses have found benefits from community pharmacist interventions for the management of CVD risk factors such as hypertension and dyslipidemia. Blenkinsopp et $\mathrm{a}^{41}$ analyzed the results of two RCTs, two observational studies, and two uncontrolled studies. The 
first RCT (the Canadian Study of Cardiovascular Risk Intervention by Pharmacists trial) compared the efficacy of pharmacist intervention (which consisted of interviewing patients and referring them to physicians, listing any risk factors, medications, and recommendations) on cholesterol risk management in high-risk coronary heart disease patients with usual pharmacy care. In the second RCT (undertaken in the USA), patients were identified from pharmacy records and invited to participate. They completed a questionnaire, underwent blood testing, and were referred to physicians according to the guidelines. Outcome measures included lipid profiles, addition or modification of lipid-lowering therapy, patient satisfaction, and quality of life. In the Study of Cardiovascular Risk Intervention by Pharmacists trial, 58\% of the intervention group showed improvements in lipid profile and had lipid-lowering therapy started or modified, compared with $30 \%$ in the control group. In the American study, $32 \%$ of the intervention group achieved target lipid levels, compared with $15 \%$ of control group participants.

The systematic review by Machado et al of 23 pharmacist interventions aimed at lowering cholesterol levels included eight studies undertaken in the community settings. ${ }^{45}$ Interventions were a combination of one or more of patient education, medication management, and lifestyle changes. ${ }^{45}$ However, most results from community pharmacy studies were excluded from meta-analysis, and those included were pooled with other settings, so this offers limited insight. The heterogeneity of settings and intervention characteristics suggested that caution is required in interpreting the meta-analysis results (although these were largely in favor of pharmacist intervention). ${ }^{46}$

The evidence provided by these studies underlines the potential of community pharmacists to improve patients' lipid management outcomes and resource utilization in this area.

Machado et al reviewed pharmacist interventions in patients with hypertension. ${ }^{27}$ The review included eight studies set in community pharmacy and reported that SBP was sensitive to pharmacist intervention (which included medication management, drug therapy monitoring, and patient education). Other outcomes assessed included DBP and adherence to treatment, but meta-analysis suggested that these outcomes were not influenced by pharmacist interventions. ${ }^{27}$ Carter et al ${ }^{47}$ conducted a meta-analysis which determined that reductions in blood pressure obtained through community pharmacist involvement were significantly better than those in usual care and had results that were at least as effective as interventions performed by pharmacists or nurses employed in general practice. This review offers a unique insight into the specific intervention components, identifying significant and independent benefits from pharmacists providing key pharmaceutical care functions such as basic education to patients, medication reconciliation, and medication recommendations to physicians. However, the article did not consider the durability of the effect/period of follow-up. Perhaps more importantly for context, this meta-analysis only considered pharmacists acting within a team-based care context, which is not currently the norm in primary care in most countries. There were quite broad eligibility criteria, with quasi-randomized trials and controlled before-after studies both included.

A more focused review of 16 RCTs by Cheema et $\mathrm{al}^{48}$ specifically explored RCTs of hypertension in community pharmacy. Eleven trials included quantitative studies used for meta-analysis $(n=2246)$ and were principally from developed countries, with the exception of a study from Thailand. These reinforce the positive findings of Carter et al, ${ }^{47}$ demonstrating a moderate pooled impact both for SBP and DBP overall, for both populations with and without $\mathrm{CV}$ health conditions. Interventions included education on hypertension, its drug treatment and lifestyle, advice on improving adherence, and the identification and reporting of drug-related problems. All eleven studies included in the meta-analysis showed significant improvements in SBP and DBP as a result of pharmacist intervention. Six of the eleven studies examined the effects on BP in patients with CVD comorbidities and the remaining five examined the effects in patients without CVD problems; the reduction was found to be significant in both cases.

Another meta-analysis by Santschi et al, ${ }^{49}$ examining pharmacist interventions for hypertension in any setting, included 39 studies, of which six trials were conducted in community pharmacies and two trials in both outpatient clinics and community pharmacies. This meta-analysis found that pharmacist interventions set in community pharmacies produced a slightly larger (but nonsignificant) reduction in blood pressure.

A review by Brown et $\mathrm{al}^{43}$ recommended regular pharmacist review to maintain the positive effects on SBP and confirmed that the most effective interventions are regular review and drug therapy modification in collaboration with the other members of the healthcare team.

Pharmacist interventions being examined to reduce CVD risk increasingly consider multifaceted and complex interventions that address multiple risk factors. The RxEACH was an RCT in 56 community pharmacies in Canada. It assessed the difference in change in CVD risk between participants who received the pharmacist intervention and 
those who received usual care (no particular intervention). Adults with high CVD risk were recruited, and the intervention consisted of medication therapy management reviews, CVD risk assessment, and education from the pharmacist. The pharmacists also prescribed medications and ordered laboratory tests to achieve therapeutic targets. The intervention group had a significantly lower CVD risk (by 21\%) than that of the control group upon completion of just a 3-month intervention. The intervention also resulted in significantly lower SBP, low-density lipoprotein (LDL) cholesterol, and $\mathrm{HbA} 1 \mathrm{c}$ and a higher smoking cessation rate. The RxEACH trial was limited by the short duration of follow-up, which is not sufficient to accurately assess smoking cessation rates and may lead to overestimated or underestimated effects of the intervention on certain parameters such as LDL, HbA1c, and blood pressure. Findings are also limited in their generalizability to other countries, as the scope of community pharmacy practice (eg, prescribing) may not be as wide as that in the study setting (Alberta, Canada). ${ }^{50}$ However, a small number of health promotion-focused interventions in other jurisdictions suggest that meaningful reductions in absolute CVD risk and multiple individual risk factors might also be achievable for pharmacists without authority to prescribe and that intervention fidelity can be maintained by pharmacists for quite complex behavioral interventions. ${ }^{51-54}$

\section{Screening for undiagnosed CVD and risk factors}

In theory, positive health gains should result if pharmacists can detect undiagnosed chronic diseases and directly manage to ensure the initiation of evidence-based therapies. A large body of evidence demonstrated the feasibility of community pharmacies as screening sites for CVDs. Willis et al reviewed 16 studies from North America, the UK, Australia, Switzerland, and Thailand. ${ }^{55}$ Two studies were RCTs, and five studies used a sequential strategy, where participants were screened noninvasively first, using validated tools, and those who appeared to be at risk underwent blood testing. Pharmacy CVD screening, which was opportunistic in most cases, identified a significant number of participants who had previously unknown CVD risk factors such as hypertension, dyslipidemia, and diabetes. The uptake of referral rate was, however, low in most studies, especially when compared with referral uptake rate in GP-initiated screening. The referral method for participants who had high CVD risk was poorly reported, but in the studies that did describe this, referral was mostly undertaken by giving participants a printout of their screening results and asking them to visit their physician.
These findings highlight the need for improving the relationship between pharmacists and physicians through better and more structured referral methods to ensure appropriate levels of referral uptake. One of the advantages associated with achieving a high uptake of referral is to reduce the cost of screening per detected case and make the screening service more financially viable.

Community pharmacy-based CVD screening has also been shown to be effective at targeting hard-to-reach groups of the population such as males, underprivileged communities, and ethnic minorities..$^{2-4}$

Pharmacist initiated-screening programs for CVDs have been successful. Several studies reported the effectiveness and acceptability of these programs that used validated tools and affordable devices such as handheld dopplers and iPhones, in identifying patients with previously undiagnosed peripheral arterial disease ${ }^{56,57}$ and atrial fibrillation. ${ }^{58,59}$ In a systematic review of pharmacy-based screening, by Ayorinde et $\mathrm{al},{ }^{60}$ it was recommended that future pharmacy-based screening studies need to compare their effectiveness and cost with screening initiatives provided by other professionals. The need for developing strategies to improve the adherence of participants to pharmacist advice was also emphasized. ${ }^{60}$

\section{Discussion}

Repeated studies and systematic reviews demonstrate the benefits of pharmacist interventions to better control CVD risk factors and the health behaviors of patients. One might surmise the studies above by saying that the weight of evidence is in favor of community pharmacist interventions as a means of reducing CV-related mortality, but it remains inconclusive. Such studies often validate the causal relationship between pharmacist intervention and intermediate patient outcomes by demonstrating the impact of the intervention on mediating outcomes such as patient knowledge, health behaviors including medication adherence, the quality of prescribing, and clinician inertia around disease management. Unfortunately, few studies have been planned or powered to determine the impact on the rates of clinically important outcomes such as CV events, mortality, or hospitalizations.

For the few studies available with clinical outcomes as primary endpoints, there are mixed results. It is important in this instance to consider the pharmacy context and the nature of the intervention.

Converting the spectrum of trial-based interventions into established and funded work practices has occurred to varying extents in a number of countries. As the range of such services expands, pharmacists will be in a better 
position to tailor their interventions to the needs of individuals by selection from screening, advice, referral, adherence advice at the time of dispensing, review of the full range of prescribed medication, and therapeutic drug monitoring for drugs such as warfarin to clinical review on referral by the patient's GP of the prescribed therapy against the diagnosis and current laboratory data. Successful implementation will be underpinned by comprehensive training for pharmacists in CVD and appropriate application of the services available.

Even within the few developed countries where most trials have been conducted, community pharmacists' scope of practice varies considerably across key areas such as rights to prescribe, order pathology tests, and administer medicines. In the same vein, pharmacy culture, public expectations of pharmacy, and the quality of medical care are also likely to vary considerably. The heterogeneity of context and interventions suggests that caution should be applied to any pooling of trial results. It might be wiser to understand the nature and context for effective and ineffective interventions so that we develop a more sophisticated understanding of how interventions should be tailored to meet the needs of given population. To this end, we need considerably more trials from developing countries - it is expected that the number of people in developing regions with diabetes in 2030 will be $69 \%$ higher than that in the 2010 figure. ${ }^{61}$ Almost one third (149 million) of a projected 439 million with diabetes globally in 2030 are anticipated to live in China and India. ${ }^{61}$ Culturally tailored approaches to diabetes care are essential, ${ }^{62}$ but we are unaware of any trials or even feasibility studies in the international literature from these and many other countries where diabetes and CVD prevalence are rapidly growing.

In some developing countries, the capacity to undertake pharmacy-based studies of CVD interventions or to implement proven programs will be limited by the absence of a strong community pharmacy network, or where pharmacies do exist, the absence of fully qualified pharmacists.

The often-interdisciplinary nature of healthcare delivery for pharmacist-delivered interventions also adds complication to the interpretation of trial results. In areas such as medication review and screening, improved health outcomes typically rely on a primary care physician to act on pharmacist recommendations or notification of a positive screening. With pharmacist intervention studies, we should be mindful therefore that we are typically examining the impact of tweaking one element of a complex care process involving multiple professionals and many health system settings that drive professional behavior. The MEDMAN trial, where GPs did not follow up on many of the pharmacist recommendations as part of a collaborative review, ${ }^{32}$ offers a salient example. The pharmacist reviews and recommendations might have produced significant health improvements if they had been implemented, but there was a widespread failure to adopt recommendations. The complexity of such interventions is often overlooked, and they are simply characterized as ineffective. It is indeed true to say that the trial did not achieve a statistically significant primary outcome in the circumstances, but it would be wrong to conclude that pharmacist reviews are therefore not worthwhile.

Taking the concept of complex interventions further, it might be that the pharmacist review offers substantial benefits for patients but that other settings within the intervention chain need to be adapted to achieve these benefits. For example, Zillich et al identified the considerable importance of a prior working relationship for the delivery of effective collaborative interventions in primary care. ${ }^{63}$ Health service trials often rely on newly established relationships between professionals to test a novel intervention; hence, the real benefit may only emerge after months or years when trust has been established and processes for collaboration have become more efficient and reliable. Health system settings can also have unintended consequences. For example, work exploring management of multimorbidity and polypharmacy in Australia suggested that remuneration systems for medication reviews might have a considerable impact on the quality of care. ${ }^{11} \mathrm{~A}$ flat payment for pharmacist reviewers was considered a disincentive by some pharmacists to spending extra time with more complex patients. Likewise, one-off payments to GPs for a medication review appeared to disincentivize commitment toward ongoing implementation of pharmacist recommendations. Several further studies identify factors at a practitioner, practice organization, and policy level, which appear to influence the quality and extent of service implementation for CVD risk reduction in community pharmacy. ${ }^{64,65}$ Embedding comprehensive process evaluations within RCTs should be a priority so that the roles of both context and intervention in delivering an outcome can be understood.

In the climate of economic rationalism that currently pervades many health systems, the need to generate evidence of improvements in terms of hard outcomes has grown considerably. This is challenging for community pharmacy intervention trials - funding requirements often mean that time frames are too short and resources are too limited to enable adequately powered studies or individual patient follow-up. Data-linkage technology provides opportunity in some healthcare systems 
to follow up patient cohorts beyond the life of the initial study. This would offer the opportunity to link trial participants with outcomes from registry and mortality databases after trial completion, but to date, this has not been widely employed for studies of CV interventions in pharmacy.

It seems inevitable that the pharmacist delivery of CVrelated services will grow internationally. The international evidence around risk reduction is widespread and compelling, even if evidence of improved clinical outcomes is less common. Pharmacists already interact regularly with individuals who have established CVD and diabetes or risk thereof, ${ }^{10}$ making it convenient for patients to have services delivered there. It adds another opportunity for care in situations where evidence-treatment gaps are widespread or where medical workforce shortages are entrenched - including in rural and remote areas of developed countries and much of the developing world. Community pharmacists also have the necessary infrastructure, skills, and ethical background to deliver high-quality services, however. The importance of providing ever more evidence of improved clinical outcomes from these interventions will be the influence it may wield in terms of ensuring adequate third-party funding and acceptance by all stakeholders and consequent facilitation of benefits for both individual patients and entire populations.

\section{Disclosure}

The authors report no conflicts of interest in this work.

\section{References}

1. Lozano R1, Naghavi M, Foreman K, et al. Global and regional mortality from 235 causes of death for 20 age groups in 1990 and 2010: a systematic analysis for the Global Burden of Disease Study 2010. Lancet. 2012;380(9859):2095-2128.

2. Yusuf S, Hawken S, Ônpuu S, et al. Effect of potentially modifiable risk factors associated with myocardial infarction in 52 countries (the INTERHEART study): case-control study. Lancet. 2004;364(9438):937-952.

3. Capewell S, O'Flaherty M. What explains declining coronary mortality? Lessons and warnings. Heart. 2008;94(9):1105-1108.

4. Egan BM, Li J, Small J, Nietert PJ, Sinopoli A. The growing gap in hypertension control between insured and uninsured adults: National Health and Nutrition Examination Survey 1988 to 2010. Hypertension. 2014;64(5):997-1004.

5. Janus ED, Bunker SJ, Kilkkinen A, et al. Prevalence, detection and drug treatment of hypertension in a rural Australian population: the Greater Green Triangle Risk Factor Study 2004-2006. Intern Med J. 2008;38(12):879-886.

6. Janus ED, Tideman PA, Dunbar JA, et al. Dyslipidaemia in rural Australia: prevalence, awareness, and adherence to treatment guidelines in the Greater Green Triangle Risk Factor Study. Med J Aust. 2010;192(3):127-132.

7. Jha V, Garcia-Garcia G, Iseki K, et al. Chronic kidney disease: global dimension and perspectives. Lancet. 2013;382(9888):260-272.

8. Jhund PS, Tavazzi L. Has the 'epidemic' of heart failure been replaced by a tsunami of co-morbidities? Eur J Heart Fail. 2016;18(5):500-502.
9. Cerasuolo JO, Montero-Odasso M, Ibañez A, Doocy S, Lip GY, Sposato LA. Decision-making interventions to stop the global atrial fibrillationrelated stroke tsunami. Int J Stroke. 2017;12(3):222-228.

10. Mc Namara KP, Dunbar JA, Philpot B, Marriott JL, Reddy P, Janus ED. Potential of pharmacists to help reduce the burden of poorly managed cardiovascular risk. Aust J Rural Health. 2012;20(2):67-73.

11. Mc Namara KP, Breken BD, Alzubaidi HT, et al. Health professional perspectives on the management of multimorbidity and polypharmacy for older patients in Australia. Age Ageing. 2017;46(2):291-299.

12. Hoffmann TC, Légaré F, Simmons MB, et al. Shared decision making: what do clinicians need to know and why should they bother? Med J Aust. 2014;201(1):35-39.

13. Clark C, Smith L, Cloutier L, et al. Allied health professional-led interventions for improving control of blood pressure in patients with hypertension: a Cochrane systematic review and meta-analysis. $J$ Hypertens. 2015;33(Suppl 1):e44.

14. Fahey T, Schroeder K, Ebrahim S. Educational and organisational interventions used to improve the management of hypertension in primary care: a systematic review. Br J Gen Pract. 2005;55(520):875-882.

15. Machado M, Bajcar J, Guzzo GC, Einarson TR. Sensitivity of patient outcomes to pharmacist interventions. Part II: systematic review and meta-analysis in hypertension management. Ann Pharmacother. 2007;41(11):1770-1781.

16. Morgado MP, Morgado SR, Mendes LC, Pereira LJ, Castelo-Branco M. Pharmacist interventions to enhance blood pressure control and adherence to antihypertensive therapy: review and meta-analysis. $\mathrm{Am}$ J Health Syst Pharm. 2011;68(3):241-253.

17. Aguiar PM, Brito GC, Correr CJ, Lyra Júnior DP, Storpirtis S. Exploring the quality of systematic reviews on pharmacist interventions in patients with diabetes: an overview. Ann Pharmacother. 2014;48(7):887-896.

18. Antoine SL, Pieper D, Mathes T, Eikermann M. Improving the adherence of type 2 diabetes mellitus patients with pharmacy care: a systematic review of randomized controlled trials. BMC Endocr Disord. 2014;14:53.

19. Bolen SD, Chandar A, Falck-Ytter C, et al. Effectiveness and safety of patient activation interventions for adults with type 2 diabetes: systematic review, meta-analysis, and meta-regression. J Gen Intern Med. 2014;29(8):1166-1176.

20. Gorman KM, Foster AD, Kaspin LC, Kindermann SL, Miller RM. PDB91 Systematic review of diabetes disease management interventions. Value Health. 2012;15(4):A187.

21. Hassali MA, Nazir SU, Saleem F, Masood I. Literature review: pharmacists' interventions to improve control and management in type 2 diabetes mellitus. Altern Ther Health Med. 2015;21(1):28-35.

22. Hussein M, Brown LM. PDB59 Cost-effectiveness analysis of medication therapy management in patients with type 2 diabetes in community pharmacy/ambulatory care settings: results from a decisionanalytic Markov model. Value Health. 2012;15(4):A181.

23. Pousinho S, Morgado M, Falcão A, Alves G. Pharmacist interventions in the management of type 2 diabetes mellitus: a systematic review of randomized controlled trials. J Manag Care Spec Phar. 2016;22(5):493-515.

24. Cranor CW, Bunting BA, Christensen DB. The Asheville Project: long-term clinical and economic outcomes of a community pharmacy diabetes care program. JAm Pharm Assoc (Wash). 2003;43(2):173-184.

25. Clifford RM, Davis WA, Batty KT, Davis TM; Fremantle Diabetes Study. Effect of a pharmaceutical care program on vascular risk factors in type 2 diabetes. The Fremantle Diabetes Study. Diabetes Care. 2005;28(4):771-776.

26. Greer N, Bolduc J, Geurkink E, et al. Pharmacist-led chronic disease management: a systematic review of effectiveness and harms compared with usual care. Ann Intern Med. Epub 2016 Apr 26.

27. Machado M, Bajcar J, Guzzo GC, Einarson TR. Sensitivity of patient outcomes to pharmacist interventions. Part I: systematic review and meta-analysis in diabetes management. Ann Pharmacother. 2007;41(10): 1569-1582. 
28. Kaczorowski J, Chambers LW, Dolovich L, et al. Improving cardiovascular health at population level: 39 community cluster randomised trial of Cardiovascular Health Awareness Program (CHAP). BMJ. 2011;342:d442.

29. Roughead EE, Barratt JD, Ramsay E, et al. Collaborative home medicines review delays time to next hospitalization for warfarin associated bleeding in Australian war veterans. J Clin Pharm Ther. 2011;36(1):27-32.

30. Altowaijri A, Phillips CJ, Fitzsimmons D. A systematic review of the clinical and economic effectiveness of clinical pharmacist intervention in secondary prevention of cardiovascular disease. J Manag Care Pharm. 2013;19(5):408-416.

31. Bouvy ML, Heerdink ER, Urquhart J, Grobbee DE, Hoe AW, Leufkens HG. Effect of a pharmacist-led intervention on diuretic compliance in heart failure patients: a randomized controlled study. J Card Fail. 2003;9(5):404-411.

32. Holland R, Brooksby I, Lenaghan E, et al. Effectiveness of visits from community pharmacists for patients with heart failure: HeartMed randomised controlled trial. BMJ. 2007;334(7603):1098.

33. Ara S. A literature review of cardiovascular disease management programs in managed care populations. J Manag Care Pharm. 2004;10(4):326-344.

34. Cai H, Dai H, Hu Y, Yan X, Xu H. Pharmacist care and the management of coronary heart disease: a systematic review of randomized controlled trials. BMC Health Serv Res. 2013;13:461.

35. Lowrie R, Mair FS, Greenlaw N, et al. Pharmacist intervention in primary care to improve outcomes in patients with left ventricular systolic dysfunction. Eur Heart J. 2012;33(3):314-324.

36. Cheng WH, Manzoor BS, Cavallari L, et al. Quality of pharmacistmanaged anticoagulation therapy in long-term ambulatory settings: a systematic review. Value Health. 2014;17(3):A126-A127.

37. Saokaew S, Permsuwan U, Chaiyakunapruk N, Nathisuwan S, Sukonthasarn A. Effectiveness of pharmacist-participated warfarin therapy management: a systematic review and meta-analysis. JThromb Haemost. 2010;8(11):2418-2427.

38. Harrison J, Shaw JP, Harrison JE. Anticoagulation management by community pharmacists in New Zealand: an evaluation of a collaborative model in primary care. Int J Pharm Pract. 2015;23(3):173-181.

39. Basaraba JE, Picard M, George-Phillips K, Mysak T. Pharmacists as care providers for stroke patients: a systematic review. Can J Neurol Sci. 2015;45(1):49-55.

40. Salgado TM, Moles R, Benrimoj SI, Fernandez-Llimos F. Pharmacists' interventions in the management of patients with chronic kidney disease: a systematic review. Nephrol Dial Transplant. 2012;27(1):276-292.

41. Blenkinsopp A, Anderson C, Armstrong M. Systematic review of the effectiveness of community pharmacy-based interventions to reduce risk behaviours and risk factors for coronary heart disease. J Public Health Med. 2003;25(2):144-153.

42. Saba M, Diep J, Saini B, Dhippayom T. Meta-analysis of the effectiveness of smoking cessation interventions in community pharmacy. $J$ Clin Pharm Ther. 2014;39(3):240-247.

43. Brown TJ, Todd A, O'Malley C, et al. Community pharmacy-delivered interventions for public health priorities: a systematic review of interventions for alcohol reduction, smoking cessation and weight management, including meta-analysis for smoking cessation. BMJ Open. 2016;6(2):e009828.

44. Brown TJ, Todd A, O'Malley C, et al. Community pharmacy interventions for public health priorities: a systematic review of community pharmacy-delivered smoking, alcohol and weight management interventions. Public Health Research, No. 4.2. 2016.

45. Machado M, Nassor N, Bajcar JM, Guzzo GC, Einarson TR. Sensitivity of patient outcomes to pharmacist interventions. Part III: systematic review and meta-analysis in hyperlipidemia management. Ann Pharmacother. 2008;42(9):1195-1207.
46. Charrois TL, Tsuyuki RT. Comment: sensitivity of patient outcomes to pharmacist interventions. Part III: systematic review and meta-analysis in hyperlipidemia management. Ann Pharmacother. 2009;43(4):793-794.

47. Carter BL, Rogers M, Daly J, Zheng S, James PA. The potency of teambased care interventions for hypertension: a meta-analysis. Arch Intern Med. 2009;169(19):1748-1755.

48. Cheema E, Sutcliffe P, Singer DR. The impact of interventions by pharmacists in community pharmacies on control of hypertension: a systematic review and meta-analysis of randomized controlled trials. Br J Clin Pharmacol. 2014;78(6):1238-1247.

49. Santschi V, Chiolero A, Colosimo AL, et al. Improving blood pressure control through pharmacist interventions: a meta-analysis of randomized controlled trials. J Am Heart Assoc. 2014;3(2):e000718.

50. Tsuyuki RT, Al Hamarneh YN, Jones CA, Hemmelgarn BR. The Effectiveness of pharmacist interventions on cardiovascular risk: the multicenter randomized controlled RxEACH trial. J Am Coll Cardiol. 2016;67(24):2846-2854.

51. Mc Namara K, George J, O'Reilly S, et al. Engaging community pharmacists in the primary prevention of cardiovascular disease: protocol for the Pharmacist Assessment of Adherence, Risk and Treatment in Cardiovascular Disease (PAART CVD) pilot study. BMC Health Serv Res. 2010;10:264.

52. McNamara KP, O’Reilly SL, Dunbar JA, et al. A pilot study evaluating multiple risk factor interventions by community pharmacists to prevent cardiovascular disease: The PAART CVD pilot project. Ann Pharmacother. 2012;46(2):183-191.

53. McNamara KP, O'Reilly SL, George J, et al. Intervention fidelity for a complex behaviour change intervention in community pharmacy addressing cardiovascular disease risk. Health Educ Res. 2015;30(6):897-909.

54. DiDonato KL, May JR, Lindsey CC. Impact of wellness coaching and monitoring services provided in a community pharmacy. $J$ Am Pharm Assoc (2003). 2013;53(1):14-21.

55. Willis A, Rivers P, Gray LJ, Davies M, Khunti K. The effectiveness of screening for diabetes and cardiovascular disease risk factors in a community pharmacy setting. PLoS One. 2014;9(4):e91157.

56. Winfrey C, Wortman S, Frede S, Kunze N, Conrad WF, Heaton PC. Pharmacist-initiated peripheral arterial disease screening program in a community pharmacy setting. JAm Pharm Assoc. 2011;51(3):373-377.

57. Zerumsky K, Steinmetz KL, Handler SM, Rodriguez EG. Pharmacist detection of peripheral arterial disease through the use of a handheld Doppler. Pharmacotherapy. 2005;25(6):797-802.

58. Twigg MJ, Thornley T, Scobie N. Identification of patients with atrial fibrillation in UK community pharmacy: an evaluation of a new service. Int J Clin Pharm. 2016;38(4):784-787.

59. Lowres N, Krass I, Neubeck L, et al. Atrial fibrillation screening in pharmacies using an iPhone ECG: a qualitative review of implementation. Int J Clin Pharm. 2015;37(6):1111-1120.

60. Ayorinde AA, Porteous T, Sharma, P. Screening for diseases in community pharmacies. Int J Pharm Pract. 2013;21:349-361.

61. Shaw JE, Sicree RA, Zimmet PZ. Global estimates of the prevalence of diabetes for 2010 and 2030. Diabetes Res Clin Pract. 2010;87(1):4-14.

62. Alzubaidi H, Mc Namara K, Browning C. Time to question diabetes self-management support for Arabic-speaking migrants: exploring a new model of care. Diabet Med. 2017;34(3):348-355.

63. Zillich AJ, McDonough RP, Carter BL, Doucette WR. Influential characteristics of physician/pharmacist collaborative relationships. Ann Pharmacother. 2004;38(5):764-770.

64. Puspitasari HP, Costa DS, Aslani P, Krass I. An explanatory model of community pharmacists' support in the secondary prevention of cardiovascular disease. Res Social Adm Pharm. 2016;12(1):104-118.

65. Mc Namara KP, Peterson GM, Hughes J, et al. Cardiovascular disease risk assessment in Australian Community Pharmacy. Heart Lung Circ. 2017;26(7):667-676. 
Integrated Pharmacy Research and Practice is an international, peer-reviewed, open access, online journal, publishing original research, reports, reviews and commentaries on all areas of academic and professional pharmacy practice. This journal aims to represent the academic output of pharmacists and pharmacy practice with particular focus on integrated care. All papers are carefully peer reviewed to ensure the highest standards as well as ensuring that we are informing and stimulating pharmaceutical professionals. The manuscript management system is completely online and includes a very quick and fair peer-review system, which is all easy to use. Visit http://www.dovepress.com/ testimonials.php to read real quotes from published authors.

Submit your manuscript here: http://www.dovepress.com/integrated-pharmacy-research-and-practice-journal 